Article

\title{
Lactobacillus plantarum outperforms Enterococcus faecium on anti-obesity possibly through the differences in gut dysbiosis attenuation, despite the similar anti-inflammatory properties.
}

\author{
Thunnicha Ondee ${ }^{1}$, Krit Pongpirul ${ }^{1,2,3,{ }^{*}}$, Kantima Janchot ${ }^{1}$, Suthicha Kanacharoen ${ }^{4}$, Thanapat Lertmongkolaksorn \\ , Lampet Wongsaroj ${ }^{6}$, Naraporn Somboonna ${ }^{6,7}$, Natharin Ngamwongsatit ${ }^{8}$ and Asada Leelahavanichkul ${ }^{9,10,11, *}$ \\ 1 Department of Preventive and Social Medicine, Faculty of Medicine, Chulalongkorn University, Bangkok \\ 10330, Thailand; thunnichaon@yahoo.com \\ 2 Department of International Health, Johns Hopkins Bloomberg School of Public Health, Baltimore, MD, \\ USA \\ 3 Bumrungrad International Hospital, Bangkok 10110, Thailand \\ 4 Department of Biology, Krieger School of Arts and Sciences, Johns Hopkins University, Baltimore, MD, \\ USA \\ 5 Research Management and Development Division, Office of the President, Mahidol University, Nakhon \\ Pathom 73170, Thailand \\ 6 Department of Microbiology, Faculty of Science, Chulalongkorn University, Bangkok 10330, Thailand \\ 7 Microbiome Research Unit for Probiotics in Food and Cosmetics, Chulalongkorn University, Bangkok \\ 10330, Thailand \\ 8 Department of Clinical Sciences and Public Health, Faculty of Veterinary Science, Mahidol University, Na- \\ khon Pathom 73170, Thailand \\ 9 Department of Microbiology, Faculty of Medicine, Chulalongkorn University, Bangkok 10330, Thailand \\ 10 Translational Research in Inflammation and Immunology Research Unit (TRIRU), Department of Microbiol- \\ ogy, Chulalongkorn University, Bangkok, 10330 Thailand \\ 11 Nephrology Unit, Department of Medicine, Faculty of Medicine, Chulalongkorn University, Bangkok 10330, \\ Thailand \\ * Correspondence: KP: doctorkrit@gmail.com; AL: a_leelahavanit@yahoo.com
}

\begin{abstract}
Fat reduction and anti-inflammation are commonly claimed properties of probiotics. Lactobacillus plantarum and Enterococcus faecium were tested in high fat-induced obesity mice and in vitro experiments. After 16 weeks of probiotics, L. plantarum outperforms E. faecium on the antiobesity property as indicated by body weight, regional fat accumulation, serum cholesterol, inflammatory cytokines (in blood and colon tissue), and gut barrier defect (FITC-dextran assay). With fecal microbiome analysis, L. plantarum but not E. faecium reduced fecal abundance of pathogenic Proteobacteria without an alteration in total Gram-negative bacteria when compared with non-probiotics obese mice. With palmitic acid induction, the condition media from both probiotics similarly attenuated supernatant IL-8, improved enterocyte integrity and down-regulated cholesterol absorptionassociated genes in Caco-2 cell (an enterocyte cell line) and reduced supernatant cytokines (TNF- $\alpha$ and IL-6) with normalization of cell energy status (extracellular flux analysis) in bone-marrow-derived macrophages. Because the anti-inflammatory effect of the condition media of both probiotics on palmitic acid-activated enterocytes was neutralized by amylase, the active anti-inflammatory molecules might, partly, be exopolysaccharides. As L. plantarum out-performed E. faecium in antiobesity property, possibly through the reduced fecal Proteobacteria, with a similar anti-inflammatory exopolysaccharide; L. plantarum is a potentially better option for anti-obesity than E. faecium.
\end{abstract}

Keywords: Probiotics; Dysbiosis; Obesity; High Fat Diet; Lactobacillus plantarum; Enterococcus faecium

\section{Introduction}


Obesity, a major health problem around the world1, is linked to diabetes, dyslipidemia, and cardiovascular disease, all of which can cause serious consequences in critically ill patients [1]. Obesity-induced chronic inflammation leads to atherosclerosis, a major vascular consequence of obesity [2]. Despite the high prevalence of obesity-induced inflammation, the pathogenesis of this condition is still uncertain. It is thought to be a combination of several mechanisms, such as hypertrophic adipocyte hypoxia and apoptosis [3,4], reduced adiponectin with leptin elevation [5], saturated fatty acid-induced inflammation and mitochondria dysfunction6, and metabolic endotoxemia from gut barrier defect [7]. Among these, the immune responses against endotoxins may lead to the most potent response because immune activation by the organism's pathogen-associated molecular patterns (PAMPs) is naturally more severe than the response towards the host cell's damage-associated molecular patterns (DAMPs) [8]. Perhaps, toll-like receptor 4 (TLR-4) activation by the lipid component, especially saturated fatty acid [9], might induce intestinal inflammation that leads to gut barrier defect and metabolic endotoxemia with systemic inflammation [10]. Indeed, endotoxin (lipopolysaccharide; LPS) has a molecular weight of $10-100 \mathrm{kDa}$ and is found in the cell walls of Gram-negative bacteria, which are the most abundant organisms of gut microbiota [11]. The molecules with a molecular weight (MW) of more than $600 \mathrm{Da}$ are unable to pass across the intestinal tight junction barrier under normal circumstances.

Obesity and a high-fat diet (HFD) produce gut dysbiosis [12] (an alteration of organisms in the intestine [13]), which increases gut-mucosal damage to the point where highMW molecules, such as LPS, can be directly translocated into the liver and circulatory system [14,15]. Non-alcoholic fatty liver disease (NAFLD), also called obesity-induced steatohepatitis, is a serious consequence of obesity that is exacerbated by the presence of LPS in blood circulation [16].

However, gut-leakage in obesity [14] is attenuated by host-beneficial probiotics [1719], partly through the improved intestinal integrity by some anti-inflammatory substances [20-24]. Among all probiotic treatments against obesity, Lactobacillus plantarum and Enterococcus faecium are lactic acid-producing bacteria that are frequently used [25], partly due to the well-tolerance against acid and bile in the intestine26 and the possible beneficial synergy with combined probiotics [27]. However, some strains of E. faecium may be pathogenically harmful to humans as causing several sites of infection (bacteremia, endocarditis, urinary tract infection) with the common anti-microbial resistance to many commonly used antibiotics [28]. Perhaps, E. faecium should be used if there is a prominent anti-obesity property and a comparison between the possible harmfulness versus the effectiveness of E. faecium are in need. On the other hand, the adverse effect of Lactobacilli probiotics is not prominent and is mostly reported in the immune-compromised host [29]. Recently, L. plantarum and E. faecium with the in vitro probiotic properties have been isolated from the Thai population and the Thai-isolated probiotics might be different from probiotics from the Caucasians due to the possible influence of some specific characteristics (ethnics, diets, climate, and co-evolution impact) [30,31]. Hence, L. plantarum and E. faecium were used in a mouse model with saturated fat-induced obesity and were also tested in the in vitro experiments.

\section{Materials and Methods}

\subsection{Animals and animal model}

The protocol of animal care and use was approved by the Institutional Animal Care and Use Committee of the Faculty of Medicine, Chulalongkorn University, Bangkok, Thailand (SST 025/2563) in compliance with US National Institutes of Health standards. As such, 8-week-old male C57BL/6 mice were purchased from Nomura Siam 
(Pathumwan, Thailand). The regular mouse diet was a standard laboratory chow containing fat $(4.5 \% \mathrm{w} / \mathrm{w})$ with energy content calculated at $3.04 \mathrm{kcal} / \mathrm{g}$ (Mouse Feed Food No.082, C.P. Company, Bangkok, Thailand), while the high-fat diet (HFD) containing fat, mostly from lard $(60 \% \mathrm{w} / \mathrm{w})$, with energy content calculated at $8.64 \mathrm{kcal} / \mathrm{g}$ [32] were used. L. plantarum dfa1 or E. faecium (Chulalongkorn University, Bangkok, Thailand) daily at $1 \times 10^{8}$ colonies forming unit (CFU) in $0.5 \mathrm{ml}$ phosphate buffer solution (PBS) or PBS alone for 16 weeks before sacrificing with cardiac puncture under isoflurane anesthesia. $L$. plantarum dfa1 and E. faecium were isolated from the feces of volunteers participating in the RDG 6150124 project of Thailand Science Research and Innovation (TSRI) of the Faculty of Medicine, Chulalongkorn University; their functional properties were studied in vitro. Two probiotics were stored at $-80{ }^{\circ} \mathrm{C}$ in MRSc broth for L. plantarum dfa1 and BHI broth for E. faecium with $20 \%$ glycerol. The probiotics were propagated each in broth for $24 \mathrm{~h}$ at $37^{\circ} \mathrm{C}$, using $1 \%$ inoculum, and then sub-cultured at $37^{\circ} \mathrm{C}$ for $24 \mathrm{~h}$ before use in each experiment. Livers and skin were snap-frozen in liquid nitrogen and kept at $-80{ }^{\circ} \mathrm{C}$ before use. Feces from all parts of the colon were combined and collected for microbiome analysis and were used to measure fecal burdens of L. plantarum dfa1 or E. faecium using polymerase chain reaction (PCR).

\subsection{Gut leakage measurement}

Gut permeability was determined by fluorescein isothiocyanate dextran (FITC-dextran) assay, endotoxemia, and immunofluorescent detection of a tight junction protein (zona occludens-1; ZO-1) following previous publications [33-35]. As such, FITC-dextran, a nonabsorbable molecule with $4.4 \mathrm{kDa}$ molecular mass (Sigma-Aldrich, St. Louis, MO, USA) at $12.5 \mathrm{mg}$ per mice was orally administered at $3 \mathrm{~h}$ before the detection of FITCdextran in serum by Fluorospectrometer (NanoDrop 3300; ThermoFisher Scientific, Wilmington, DE, USA). Serum endotoxin (LPS) was measured by HEK-Blue LPS Detection (InvivoGen, San Diego, CA, USA) and the data were recorded as 0 when LPS values were less than $0.01 \mathrm{EU} / \mathrm{ml}$ because of the limited lower range of the standard curve.

\subsection{Analysis of mouse samples from blood, organs, and feces}

After fasting for 12 hours after free access to drinking water, lipid profiles were measured by the quantification assay for triglyceride (TG), total cholesterol (Sigma-Aldrich, St. Louis, MO, USA), low- and high-density lipoprotein cholesterol (LDL and HDL) (Crystal Chem Inc., Downers Grove, IL, USA). Liver damage and serum cytokines were determined by EnzyChrom Alanine Transaminase assay (EALT-100, BioAssay, Hayward, CA, USA) and enzyme-linked immunosorbent assays (ELISA) for mouse cytokines (Invitrogen, Carlsbad, CA, USA), respectively. For histology, paraffin-embedded sections (4 $\mu \mathrm{m}$ thick) stained by Hematoxylin and Eosin (H\&E) from 10\% formalin-fixed samples were evaluated. The scoring system of obesity-induced liver damage was used like the following; steatosis (0-3), lobular inflammation (0-3), and hepatocellular ballooning degeneration (0-2) [36]. The thickness of subcutaneous fat was determined following a previous publication37. For the detection of lipids in the liver, livers were sonicated (High-Intensity Ultrasonic Processor, Newtown, CT, USA) in $500 \mu$ l of ice-cold PBS containing protease inhibitor Cocktail (I3786) (Sigma-Aldrich, St. Louis, MO, USA) and measured lipids from the supernatant by the quantification assay for triglyceride and total cholesterol (SigmaAldrich, St. Louis, MO, USA).

In addition, oxidative stress in the liver was evaluated following a previous study [38]. Briefly, livers were homogenized in radioimmunoprecipitation assay (RIPA) with protease inhibitor Cocktail (I3786) (Sigma-Aldrich, St. Louis, MO, USA) on ice before measuring an oxidative stress molecule, malondialdehyde (MDA), (Cayman Chemical Company, Ann Arbor, MI, USA). For an anti-oxidant molecule, livers were sonicated in 2-(N-morpholino) ethanesulfonic acid (MES) buffer (Sigma-Aldrich, St. Louis, MO, USA) before the measurement of glutathione (GSH) (Cayman Chemical Company, Ann Arbor, 
MI, USA) from the supernatant. Moreover, for cytokine detection in colon tissue, the samples were weighed, cut, thoroughly sonicated (High-Intensity Ultrasonic Processor, Newtown, CT, United States) in $500 \mathrm{ml}$ of ice-cold PBS containing protease inhibitor Cocktail (I3786; Sigma-Aldrich, St. Louis, MO, USA) and measured cytokines from the supernatant by ELISA (Invitrogen; ThermoFisher Scientific, Wilmington, DE, USA).

\subsection{Mouse fecal analysis}

The bacterial abundance of L. plantarum or E. faecium was evaluated by real-time polymerase chain reaction (PCR) following a previous publication10. Briefly, total DNA was extracted by a QIAamp fast DNA Stool Mini Kit (Qiagen, Hiden, Germany), as per the manufacturer's instructions. Primers for the variable regions of the $16 \mathrm{~s}$ ribosomal RNA (rRNA) gene sequence of $L$. plantarum bacteria [39] were: Lp-F, 5'AAAATCATGCGTGCGGGTAC-3'; Lp-R, 5'-ATGTTGCGTTGGCTTCGTCT-3' and for E. faecium [40] were E16S 72f, 5'-CCGAGTGCTTGCACTCAATTGG-3'; E16S 210r, 5'CTCTTATGCCATGCGGCATAAAC-3'. The constructive standard curve of L. plantarum and E. faecium was generated by the QuantStudio ${ }^{\mathrm{TM}}$ Design \& Analysis Software v1.4.3 (Thermo Fisher Scientific, Wilmington, DE, USA) using 10-fold serial dilution with bacterial concentrations ranging from 2 to 2 x105 bacteria were used to convert the PCR results into bacterial copy numbers.

\subsection{Fecal microbiome analysis}

Feces from 9 mice ( $0.25 \mathrm{~g}$ per mouse) from different cages in each experimental group were divided into three samples per group ( 3 mice per sample) before performing microbiota analysis following a previous protocol41. In short, metagenomic DNA was extracted from $0.25 \mathrm{~g}$ feces by DNeasy PowerSoil Kit (Qiagen, Maryland, USA). The Universal prokaryotic 515F (forward; (5'-GTGCCAGCMGCCGCGGTAA-3') and 806R (reverse; 5'GGACTACHVGGGTWTCTAAT-3'), with appended Illumina adapter and Golay barcode sequences, were used for 16S rRNA gene V4 library construction and sequenced using Miseq300 platform (Illumina, San Diego, Calif, USA) at Omics Sciences and Bioinformatics Center, and Microbiome Research Unit for Probiotics in Food and Cosmetics, Chulalongkorn University. Raw sequences were quality processed and operational taxonomic unit (OTU) classified following Mothur's standard operating platform procedures $[42,43]$. Bioinformatic analyses included good's coverage, alpha diversity (e.g. Chao), and beta diversity (e.g. non-metric multidimensional scaling (NMDS)). Linear discriminant analysis effect size (LEfSe) and meta-stats were also performed to determine species marker and unique representing species of the interested group, respectively $[42,44]$.

\subsection{Anti-inflammatory responses of enterocytes}

Because of the possible differential impact of L. plantarum versus E. faecium on enterocytes, the in vitro experiments using the Caco-2 enterocyte cell line were performed. Caco-2 (ATCC HTB-37) from the American Type Culture Collection (Manassas, VA, USA) were maintained in supplemented Dulbecco's modified Eagle medium (DMEM) at $37^{\circ} \mathrm{C}$ under $5 \% \mathrm{CO} 2$ and sub-cultured before use in the experiments. Then, Caco-2 cells at $1 \times 10^{6}$ cells/well were incubated with palmitic acid at $0.5 \mathrm{mM} /$ well or $100 \mathrm{ng} / \mathrm{mL}$ of lipopolysaccharide (LPS) from E. coli O26:B6 (Sigma-Aldrich, St. Louis, MO, USA) or palmitic acid with LPS (palmitic acid + LPS) with or without $5 \%(\mathrm{vol} / \mathrm{vol})$ condition media of probiotics (each strain) (the total volume was adjusted into $200 \mu \mathrm{L} /$ well by the culture media) for 24 hours before determination of supernatant IL-8 using ELISA (Quantikine Immunoassay; R\&D Systems, Minneapolis, MN, USA). For the preparation of probiotics condition media, L. plantarum or E. faecium at an OD600 of 0.1 were incubated anaerobically for 48 hours before supernatant collection by centrifugation and filtration $(0.22-\mu \mathrm{m}$ membrane filter) (Minisart; Sartorius Stedim Biotech GmbH, Göttingen, Germany). After that, the supernatant of the samples $(500 \mu \mathrm{L})$ was concentrated by speed vacuum drying at $40^{\circ} \mathrm{C}$ for 3 hours 
(Savant Instruments, Farmingdale, NY, USA), resuspended in an equal volume of DMEM, and stored at $-20^{\circ} \mathrm{C}$ until use. Because of the different enriched media for L. plantarum or E. faecium using De Man, Rogosa and Sharpe broth (MRSc) and Brain Heart Infusion broth (BHI), respectively, the media were also used as control. Additionally, to explore molecular characteristics of the effective anti-inflammatory molecules in condition media from $L$. plantarum (LpCM) or E. faecium (EfCM) against saturated fatty acid-induced enterocyte inflammation, various enzymes including; i) $\alpha$ - amylase (in $20 \mathrm{mM}$ sodium acetate with $7 \mathrm{mM}$ sodium chloride), ii) lipase (in $50 \mathrm{mM}$ Tris- $\mathrm{HCl}$ ), and iii) proteinase $\mathrm{K}$ (in $50 \mathrm{mM}$ Tris- $\mathrm{HCl}$ ) (Sigma-Aldrich, St. Louis, MO, USA) at $1 \mathrm{mg} / \mathrm{mL}$ were incubated with the 24 hour-activated Caco- 2 cells (1x104 cells/well) (palmitic acid with or without LPS) at $25^{\circ} \mathrm{C}$ (for amylase) and $37^{\circ} \mathrm{C}$ (for lipase and proteinase) for 6 hours before inactivation by heating at $100{ }^{\circ} \mathrm{C}$ for 10 minutes and supernatant IL-8 measurement by ELISA (R\&D Systems, Minneapolis, MN, USA).

\subsection{Gene expression of cholesterol absorption-associated molecules in enterocytes}

To explore the impact of the probiotic condition media on cholesterol absorption, the RNA from stimulated Caco-2 cells was prepared using FavorPrep Tissue total RNA purification Mini Kit (Favorgen Biotech Corp, Vienna, Austria) and cDNA was synthesized by cDNA Synthesis assay (Thermo Fisher Scientific, Wilmington, DE, USA) before the detection by SYBR green-based real-time PCR (Thermo Fisher Scientific, Wilmington, DE, USA). The oligonucleotide primers for the experiment were i) NPC Intracellular Cholesterol transporter-1 (NPC-1); forward 5' -TAT GGT CGC CCG AAG CA-3' and reverse 5' TGC GGT TGT TCT GGA AAT ACTG-3', ii) ATP Binding Cassette Subfamily G Member 5 (ABCG5); forward 5'-ACC CAA AGC AAG GAA CGG GAA-3' and reverse 5'-CAG CGT TCA GCA TGC CTG TGT-3', iii) ATP Binding Cassette Subfamily G Member 8 (ABCG8); forward 5'-GGG TGA GCG CAG GAG AGT CAG-3' and reverse 5'-TCA CGC TGC TTT CCA CAC AGG-3' and iv) beta-actin ( $\beta$-actin; a house-keeping gene); forward 5'-CCT GGC ACC CAG CAC AAT-3' and reverse 5'-GCC GAT CCA CAC GGA GTA CT3'. The forward and reverse primers were mixed in equal proportions and used at a final concentration of $10 \mathrm{uM}$. Samples were analyzed with QuantStudio6 Flex Real-time PCR System (Thermo Fisher Scientific, Wilmington, DE, USA) and initially preheated at $95{ }^{\circ} \mathrm{C}$ for $2 \mathrm{~min}$. Then, 45 PCR cycles were performed as follows: $95^{\circ} \mathrm{C}$ for 1 second, $60{ }^{\circ} \mathrm{C}$ for 1 minute. Melting curve profiling was performed at the end of each PCR process to confirm amplification of specific transcripts by the following steps: $95{ }^{\circ} \mathrm{C}$ for 15 seconds, cooling to $60^{\circ} \mathrm{C}$ for 1 minute, heating the sample to $95^{\circ} \mathrm{C}$ for 30 seconds, and cooling to $60{ }^{\circ} \mathrm{C}$ for 15 seconds under continuous measurement of fluorescence. The results were demonstrated in terms of relative quantitation of the comparative threshold (delta-delta $\mathrm{Ct}$ ) method ( $\left.2^{-\Delta \Delta C t}\right)$ as normalized by $\beta$-actin.

\subsection{Transepithelial electrical resistance (TEER) and enterocyte permeability}

The integrity of monolayer enterocytes in different conditions was determined by TEER using Caco-2 cells. Caco-2 cells (ATCC HTB-37) at $5 \times 10^{4}$ cells per well were seeded onto the upper compartment of 24-well Boyden chamber trans-well plate using DMEMhigh glucose supplemented with 20\% Fetal Bovine Serum (FBS), 1\% HEPES, 1\% sodium pyruvate, and $1.3 \%$ Penicillin/Streptomycin for 15 days to establish the confluent monolayer. After that, palmitic acid at $0.5 \mathrm{mM}$ / wells with or without $5 \%$ (vol/vol) LpCM or EfCM were incubated at $37^{\circ} \mathrm{C}$ under $5 \% \mathrm{CO} 2$ for $24 \mathrm{~h}$. Subsequently, TEER was measured by an epithelial volt-ohm meter (EVOM-2, World Precision Instruments, Sarasota, FL, USA) by placing the electrodes in the supernatant at the basolateral and apical chamber. The TEER value in media culture without cells was used as a blank and was subtracted from all measurements. The unit of TEER was ohm $(\Omega) \times \mathrm{cm}^{2}$. In parallel, $5 \mu \mathrm{L}$ of FITCdextran (4.4 kDa) (Sigma-Aldrich, St. Louis, MO, USA) at $10 \mathrm{mg} / \mathrm{mL}$ was added to the apical side of the trans-well chamber of the 24-hour-stimulated Caco-2 cells. Then, FITC- 
dextran from the basolateral side of the trans-well plate was measured at 3 hours after the incubation using Fluorospectrometer (NanoDrop 3300; ThermoFisher Scientific, Wilmington, DE, USA) as modified from the published protocols [45]. The concentration of FITCdextran from the basolateral side represents the severity of permeability defect of Caco-2 cells.

\subsection{Macrophage cytokines and extracellular flux analysis}

Due to the influence of probiotics on gut inflammation [46-48] and the importance of macrophages on inflammation $[49,50]$, an impact of condition media from L. plantarum or E. faecium were also tested in macrophages. Accordingly, bone marrow-derived macrophages were isolated from femurs and tibias of mice following a previous protocol [46]. Briefly, the bone marrow was collected by centrifugation at $6000 \mathrm{rpm}$ for $4^{\circ} \mathrm{C}$ and incubated for 7 days with DMEM supplemented with 10\% fetal bovine serum (FBS), 1\% penicillin/streptomycin, and 4-(2-hydroxyethyl)-1-piperazineethanesulfonic acid (HEPES) with sodium pyruvate in a humidified $5 \% \mathrm{CO} 2$ incubator at $37^{\circ} \mathrm{C}$. Conditioned media of the $\mathrm{L} 929$ cell line, containing macrophage-colony stimulating factor, at $20 \%$ weight by volume $(\mathrm{w} / \mathrm{v})$, was used to induce macrophages from the pluripotent stem cells. Then, macrophages at $1 \times 10^{5}$ cells/well were incubated for 24 hours with the control media or $0.5 \mathrm{mM}$ palmitic acid with or without $5 \%(\mathrm{v} / \mathrm{v})$ of LpCM or EfCM before the determination of supernatant cytokines by ELISA (Invitrogen) or extracellular flux analysis using Seahorse XFp Analyzers (Agilent, Santa Clara, CA, USA) with oxygen consumption rate (OCR) and extracellular acidification rate (ECAR) representing mitochondrial function (respiration) and glycolysis activity, respectively. The stimulated macrophages at $1 \times 10^{5}$ cells/well were incubated by Seahorse media (DMEM complemented with glucose, pyruvate, and L-glutamine) (Agilent, 103575-100) for 1 hour before activation by different metabolic interference compounds, including oligomycin, carbonyl cyanide-4-(trifluoromethoxy)-phenylhydrazone (FCCP) and rotenone/antimycin A, for OCR evaluation. In parallel, glycolysis stress tests were performed using glucose, oligomycin, and 2-Deoxy-d-glucose (2-DG) for ECAR measurement. The data were analyzed by Seahorse Wave 2.6 software based on the following equations: (i) maximal respiration = OCR between FCCP and rotenone/antimycin A - OCR after rotenone/antimycin A; (ii) maximal glycolysis (glycolysis capacity) $=$ ECAR between oligomycin and 2-DG - ECAR after 2-DG.

\subsection{Statistical analysis}

Mean \pm standard error (SE) was used for data presentation. The differences between groups were examined for statistical significance by one-way analysis of variance (ANOVA) followed by Tukey's analysis or Student's t-test for comparisons of multiple groups or 2 groups, respectively. All statistical analyses were performed with SPSS 11.5 software (SPSS, IL, USA) and Graph Pad Prism version 7.0 software (La Jolla, CA, USA). A p-value of $<0.05$ was considered statistically significant.

\section{Results}

\subsection{Lactobacillus plantarum outperformed Enterococcus faecium in obesity attenuation in a high-fat diet mouse model}

Both L. plantarum and E. faecium attenuated obesity in high-fat diet (HFD) mice as determined by body weight, serum lipid profile (total cholesterol and triglyceride), and visceral fat deposition in several sites (mesentery, peri-renal, retro-peritoneum, peri-gonadal, and subcutaneous fat) along with liver injury (liver weight, histological score and liver cholesterol) (Fig 1A-M) supported several studies [51,52]. In addition, both probiotics also attenuated liver enzyme (alanine transaminase), oxidative injury (reduced MDA and increased GSH), serum cytokines (TNF- $\alpha$, IL-6, and IL-10), and gut leakage (FITC-dextran assay and endotoxemia) (Fig 2A-K). However, the anti-obesity effect of L. plantarum was 
more profound than E. faecium as indicated by body weight, blood cholesterol, some regional fat deposition (mesentery, peri-renal, subcutaneous fat, and liver), oxidative stress in the liver, serum pro-inflammatory cytokines (TNF- $\alpha$ and IL-6) and gut leakage (FITCdextran assay) (Fig 1A-M and Fig 2A-K) without the difference in fecal abundance between both strains of probiotics (Fig 2L). These data supported the acceptable property of both probiotic strains in terms of the stability in the intestines (acid and bile tolerance) [26] and the possible difference in anti-obesity effect among probiotics [53]. Because i) Gramnegative bacteria in the gut is a source of endotoxin (LPS) [11] which could enter blood circulation (obesity-induced endotoxemia) 54 and ii) gut dysbiosis causes gut barrier defect is well-known $[41,46,55]$, the better effect on gut barrier defect attenuation (serum FITC-dextran assay) of L. plantarum when compared with E. faecium might be due to the different effect on gut dysbiosis.
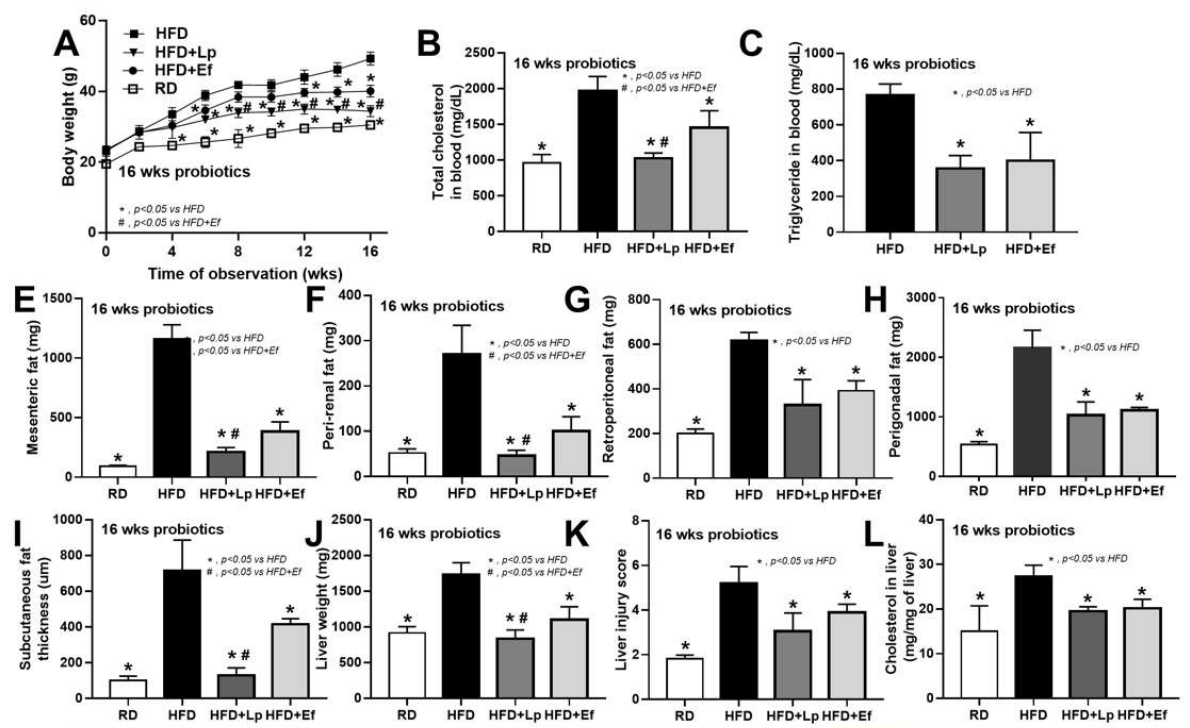

M

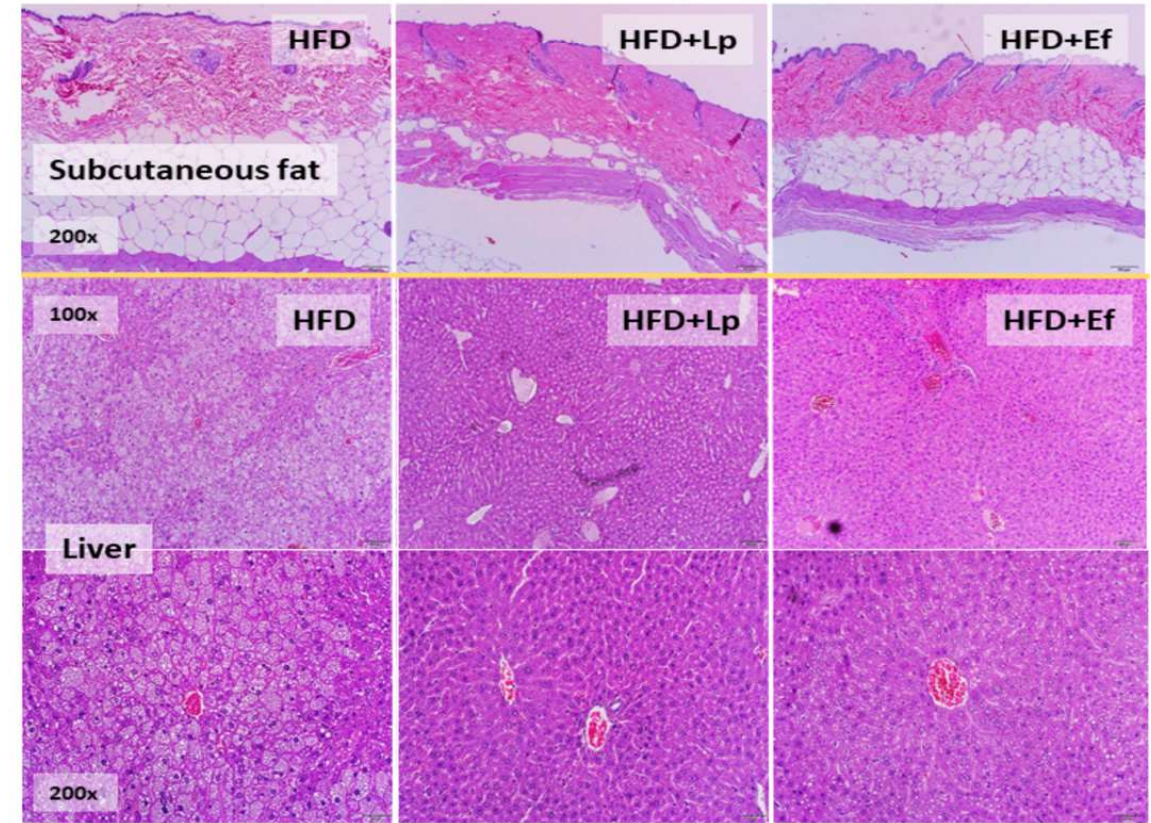

Figure 1. Characteristics of mice with regular diet (RD) or high fat diet (HFD) with or without Lactobacillus plantarum (Lp) or Enterococcus faecium (Ef) as determined by body weight (A), fasting blood lipid profile (total cholesterol and triglyceride) (B, C), adipose tissue depots in several sites 
(D-H), subcutaneous fat thickness (I), liver injury (weight, histological score and cholesterol in liver) (J-L) and the representative figures of subcutaneous fat thickness and liver (original magnification 200x) (M) were demonstrated ( $n=6-8$ / time-point or group).

A
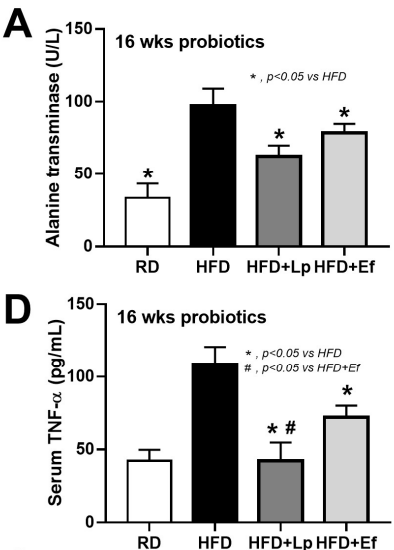

G
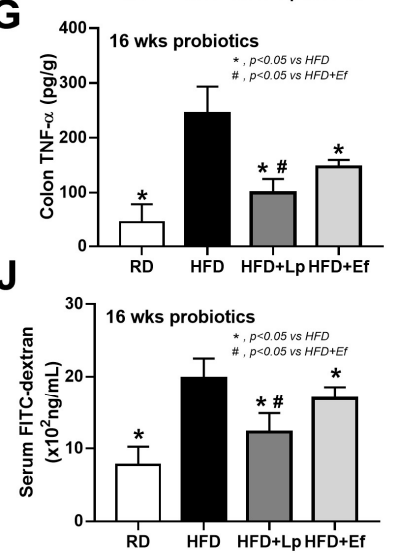
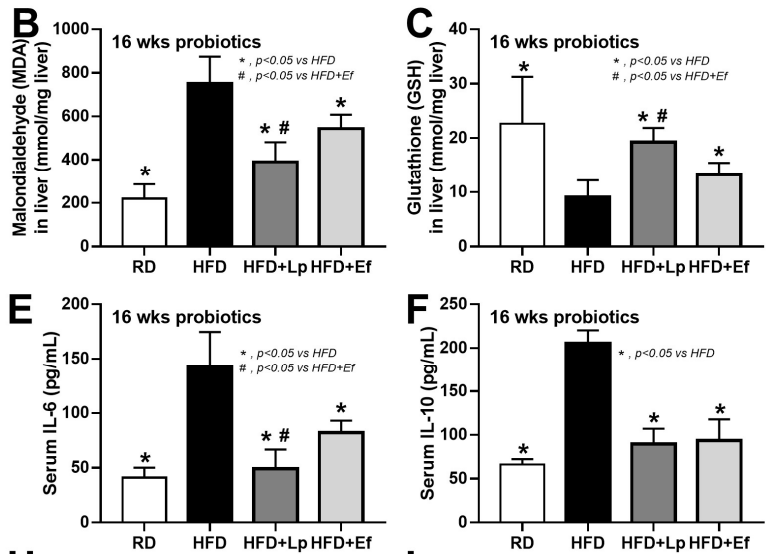

H
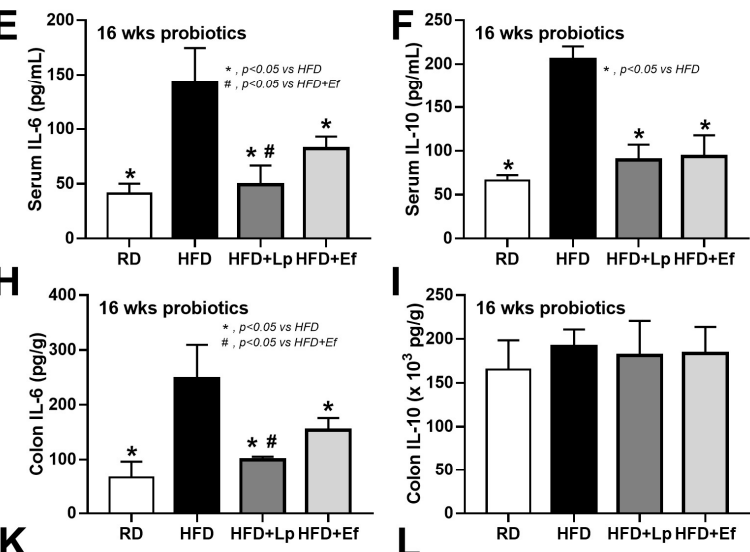

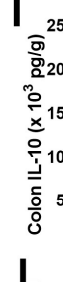
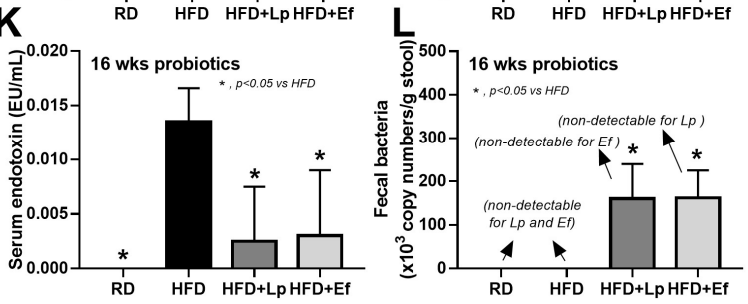

Figure 2. Characteristics of mice with regular diet (RD) or high fat diet (HFD) with or without Lactobacillus plantarum (Lp) or Enterococcus faecium (Ef) as determined by alanine transaminase (ALT) (A), oxidative stress (malondialdehyde; MDA) and anti-oxidant molecule (glutathione) in liver (B, C), serum cytokines (TNF- $\alpha$, IL-6 and IL-10) (D-F), gut leakage (FITC-dextran) (G), serum endotoxin $(\mathrm{H})$ and bacterial abundance in feces by polymerase chain reaction (I) are demonstrated $(n=6-8 /$ group).

\subsection{Lactobacillus plantarum, but not Enterococcus faecium, reduced Proteobacteria (a group of pathogenic bacteria) in feces of high-fat diet mice}

In comparison with the regular diet group, HFD increased Proteobacteria (the pathogenic Gram-negative bacteria) without causing a difference in Bacteroides (the most abundance Gram-negative bacteria in feces) and Firmicutes (the high abundance bacteria in healthy condition) with Bacteriodes spp. as the possibly unique bacteria in HFD by Linear discriminant Effect Size (LEfSe) analysis (Fig 3A-D) supporting obesity-enhanced Bacteroides bacteria as previously reported56. However, HFD did not increase the total Gram-negative bacteria in feces (Fig 3D) and the alpha diversity (Fig 3E) when compared with the regular diet group. Despite the similar Gram-negative bacterial burdens in feces of HFD compared with regular diet mice (Fig 3D), HFD induced endotoxemia (Fig 2K) implying an impact of gut dysbiosis on the intestinal permeability [57,58]. On the other hand, there was an alteration of fecal microbiome analysis with probiotic administration. In microbiome analysis on the phylum level, E. faecium did not alter the fecal abundance of Firmicutes, and Proteobacteria but reduced Bacteroides, while L. plantarum reduced 
both Firmicutes and Proteobacteria but increased Bacteroides when compared with HFD (Fig 3A-D). In comparison between L. plantarum and E. faecium administration in HFD mice, L. plantarum reduced Firmicutes and Proteobacteria but increased Bacteroides without the difference on total Gram-negative bacteria in feces (Fig 3D). The reduced Proteobacteria (pathogenic bacteria) in HFD mice after L. plantarum administration (Fig 3D) might be, at least in part, responsible for the more potent weight reduction of L. plantarum over E. faecium (Fig 1A).

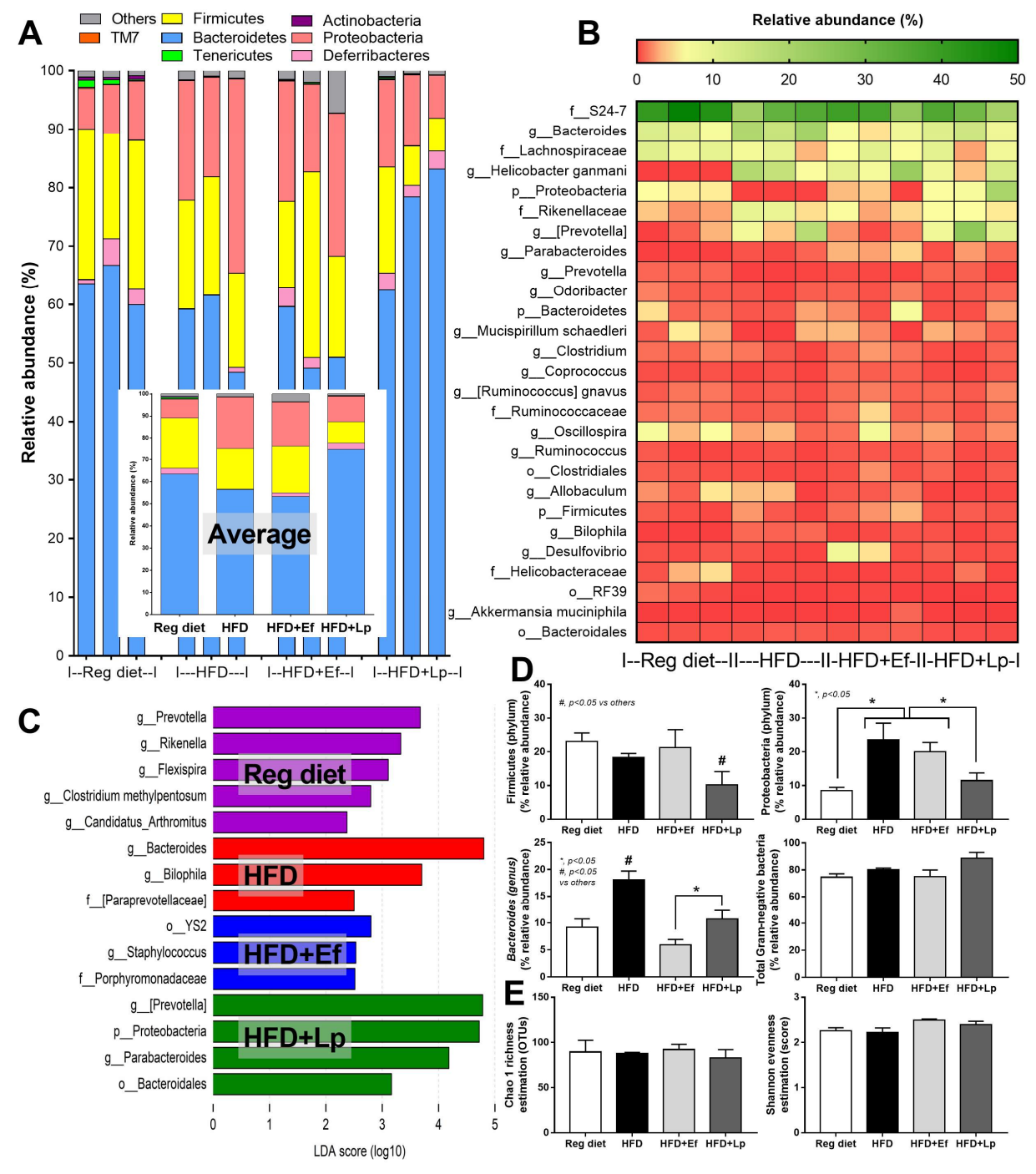

Figure 3. Gut microbiota analysis from feces of high fat diet (HFD) mice with or without Lactobacillus plantarum (Lp) or Enterococcus faecium (Ef) or mice with regular diet (RD) as determined by relative abundance of bacterial diversity at phylum (the inset graph is the average abundance) (A), and at genus (heat-map) (B), the possibly unique bacteria in each group by Linear discriminant Effect Size (LEfSe) analysis (C), graph presentation of the abundance of some groups of bacteria and total Gram-negative bacteria in feces (D) with the alpha diversity by Chao 1 richness estimation and Shannon evenness analysis (E) are demonstrated.

\subsection{Both Lactobacillus plantarum and Enterococcus faecium attenuated fatty acid-induced enterocyte inflammation through the production of carbohydrate molecules}


Because direct activation of saturated fatty acid and probiotic-producing molecules on enterocytes is possible $[59,60]$, in vitro tests using palmitic acid (a representative saturated fatty acid) activation on enterocytes with or without the condition media from probiotics were performed. Indeed, the saturated fatty acid-induced supernatant cytokine (IL-8) and induced enterocyte tight junction injury, as indicated by TEER and transepithelial FITC-dextran (Fig 4A-C), with the up-regulation of several genes that associated with cholesterol absorption (NPC-1, ABCG5, and ABCG8) (Fig 4D-F). However, the condition media of both probiotics attenuated fatty acid-induced enterocyte injury, as indicated by reduced IL-8 production, and improved enterocyte integrity (TEER and transepithelial FITC-dextran) (Fig 4A-C). Likewise, the condition media also down-regulated the cholesterol absorption-associated genes (Fig 4D-F). Because the synthesis of some anti-inflammatory molecules from probiotics might be, at least in part, responsible for the attenuation in gut barrier defect61, enzyme neutralization experiments were performed. With the activation by saturated fatty acid with or without LPS, amylase neutralized the anti-inflammatory effect of condition media of L. plantarum, while both amylase and lipase neutralized the effect of E. faecium (Fig 5A-F), implying that the characteristics of anti-inflammatory molecules of L. plantarum and E. faecium were carbohydrate and lipo-carbohydrate, respectively. The difference in active molecules of L. plantarum and E. faecium might, partly, be a factor that is responsible for the difference in anti-obesity potency.

However, these molecules from condition media of both probiotics similarly attenuated the pro-inflammatory effect of palmitic acid (saturated fatty acid) induced on macrophages as indicated by the reduction in supernatant cytokines (TNF- $\alpha$ and IL- 6 but not IL-10) and macrophage cell energy status (Fig 6A-I). While palmitic acid-enhanced glycolysis activity (basal and maximal glycolysis) and reduced mitochondrial functions (basal and maximal respiration), the conditioned media shifted the responses toward the characteristics of the control group (Fig 6D-I). Because of the prominent use of glycolysis on cytokine production in macrophages62, the reduced glycolysis and supernatant cytokines by condition media imply an impact of anti-inflammatory molecules from both probiotics.
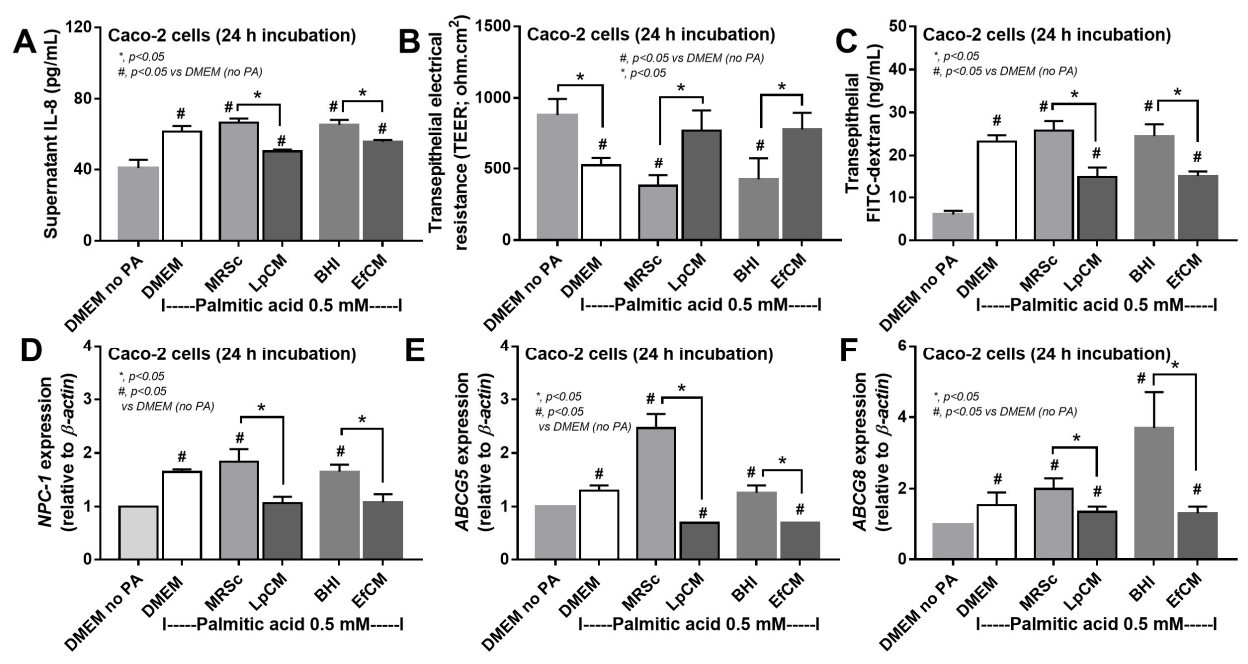

Figure 4. Characteristics of Caco-2 cells (enterocytes)with $0.5 \mathrm{mM}$ palmitic acid (PA) with or without condition media of Lactobacillus plantarum (LPCM) or Enterococcus faecium (EfCM) as indicated by supernatant IL-8 (A), transepithelial electrical resistance (TEER) (B), transepithelial FITC-dextran (C) and gene expression of cholesterol absorption associated molecules, including NPC-1 (NPC Intracellular Cholesterol Transporter 1), ABCG5 (ATP Binding Cassette Subfamily G Member 5) and ABCG8 (ATP Binding Cassette Subfamily G Member 8), (D-F) are demonstrated. Notably, MRSc (De Man, 
Rogosa and Sharpe broth) and) and BHI (Brain Heart Infusion broth) were the culture media for Lp and Ef, respectively, and independent triplicate experiments were performed for all experiments.
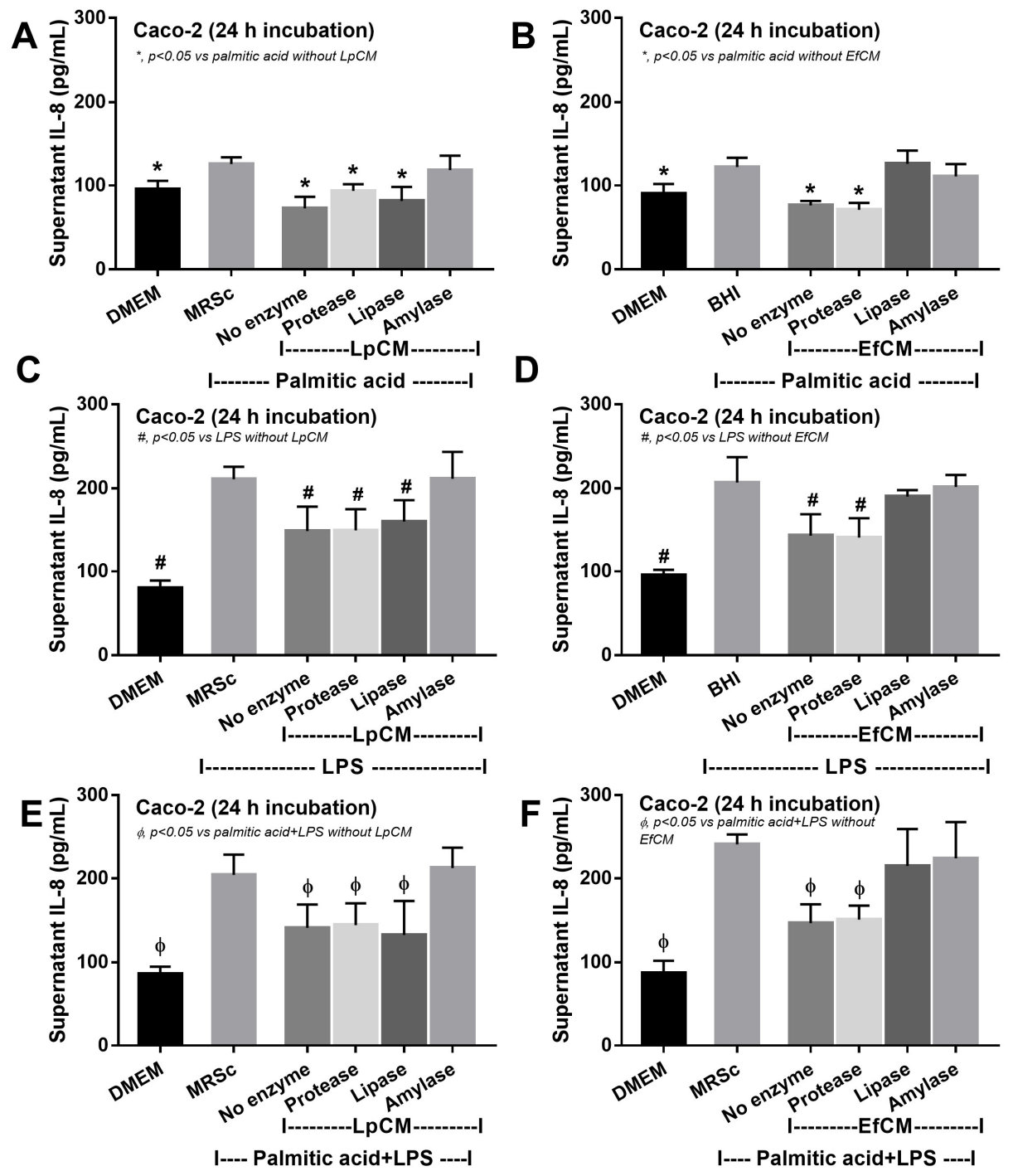

Figure 5. Supernatant IL-8 from Caco-2 cells (enterocytes)with Dulbecco's Modified Eagle Medium (DMEM; media for Caco2 cell) or probiotic condition media from Lactobacillus plantarum (LpCM) or Enterococcus faecium (EfCM) after incubated with or with out enzyme inactivation (protease, lipase and amylase) when activated by $0.5 \mathrm{mM}$ palmitic acid (A, B), $100 \mathrm{ng} / \mathrm{mL}$ of lipopolysaccharide (LPS) (C, D) or palmitic acid with LPS (E, F) are demonstrated. Notably, MRSc (De Man, Rogosa and Sharpe broth) and) and BHI (Brain Heart Infusion broth) were the culture media for Lp and Ef, respectively, and independent triplicate experiments were performed for all experiments. 

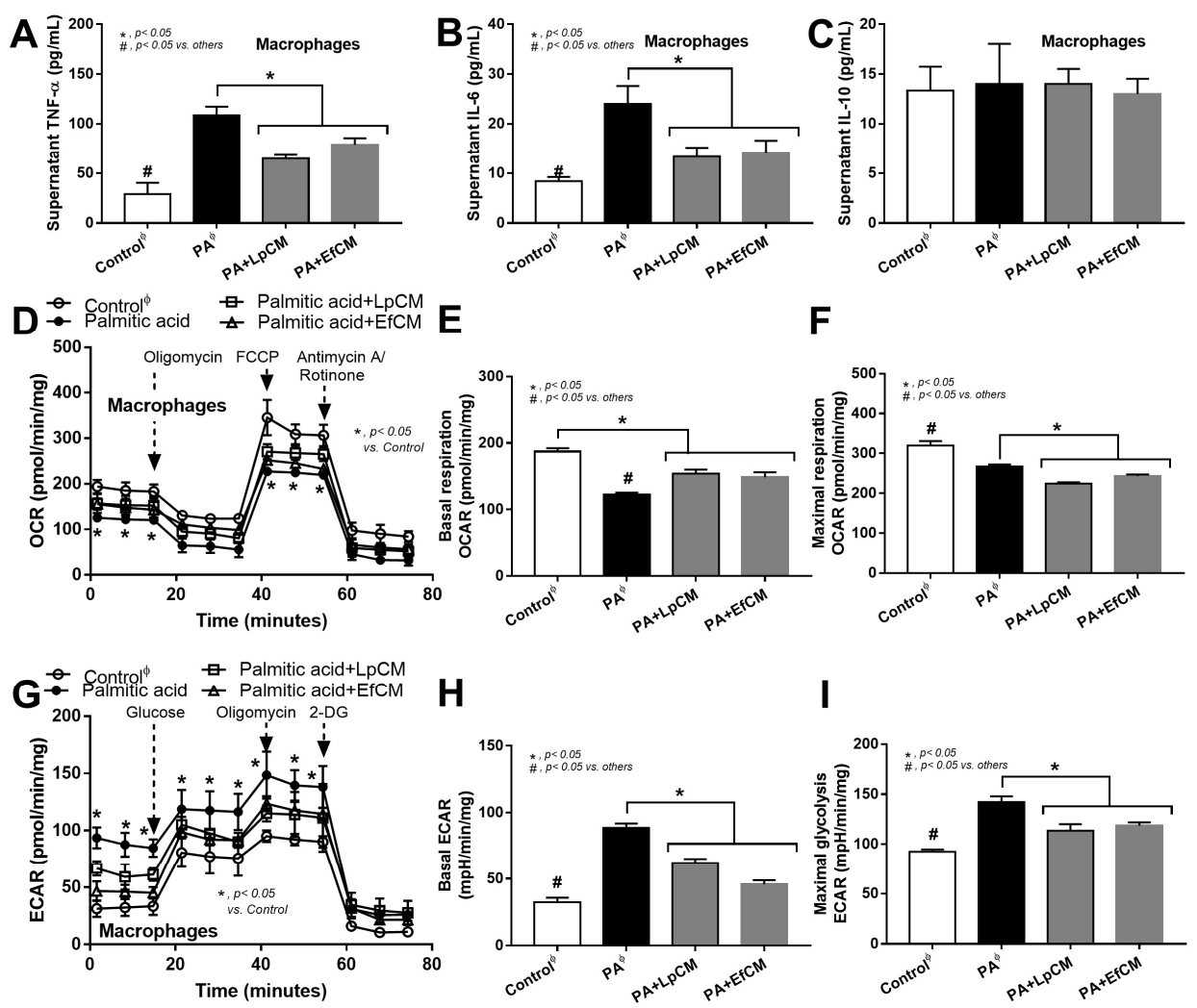

Figure 6. Characteristics of bone marrow-derived macrophages after $24 \mathrm{~h}$ activation in control or $0.5 \mathrm{mM}$ palmitic acid with or without probiotic condition media from Lactobacillus plantarum (LpCM) or Enterococcus faecium (EfCM) as indicated by supernatant cytokines (TNF- $\alpha$, IL-6 and IL-10) (A-C) and extracellular flux analysis for mitochondrial activity, including oxygen consumption rate (OCR), basal respiration and maximal respiration, and glycolysis activity, including extracellular acidification rate (ECAR), basal ECAR, maximal glycolysis, (D-I) are demonstrated (independent triplicate experiments were performed for all experiments). Notably, $\phi$ indicates the combination of data form the activation by different control, including Dulbecco's Modified Eagle Medium (DMEM; media for Caco2 cell) and bacterial culture media of Lp; MRSc (De Man, Rogosa and Sharpe broth) and for Ef; BHI (Brain Heart Infusion broth).

\section{Discussion}

Although both L. plantarum and E. faecium attenuated obesity in high-fat diet (HFD)administered mice, L. plantarum out-performed E. faecium as indicated by weight reduction, serum cytokines, and gut barrier defect, possibly through the more prominent reduction in fecal Proteobacteria with differences in the active anti-inflammatory substances.

\subsection{Dysbiosis and gut barrier defect in obese mice}

The obese mice were overweight, increased fat accumulation, hyperlipidemia, liver injury (liver weight, steatohepatitis, and elevated liver enzymes), and gut barrier defect (FITC-dextran assay and increased serum LPS). Gut barrier defect causes obesity-induced endotoxemia which is a fundamental activator leading to several complications [63], 
including liver injury and cardiovascular diseases [11], as inflammatory responses against pathogens molecules are stronger than the responses toward self-antigens [8]. As such, HFD itself increased fecal $\mathrm{pH}$, possibly through bile-production amplification, that reduces short-chain fatty acids (SCFAs) [64], increase Proteobacteria (mucosal invasive Gram-negative organisms [65,66]) but not Bacteroidetes (most prominent Gram-negative anaerobes in gut [67]) nor fecal burdens of total Gram-negative bacteria. This data supports that HFD enhances endotoxemia68 through dysbiosis-induced intestinal mucosal injury $[69,70]$, but does not increase PS burdens in the gut. Hence, the attenuation of dysbiosis and/ or gut-leakage may be a direct adjunctive treatment against obesity-induced inflammation and other complications.

\subsection{Both Lactobacillus plantarum and Enterococcus faecium attenuated obesity and gut dysbiosis}

The attenuation of obesity-induced gut dysbiosis by probiotics $[54,55,71,72]$ is explained through several mechanisms including the enhanced and effective energy in the host, reduced lipid absorption, increased SCFAs production, and promoted intestinal hormones [73]. Among several probiotics, L. plantarum and E. faecium demonstrate robust lactic acid production 26 that may alter HFD-induced dysbiosis. However, there was a different effect on fecal microbiome analysis between these probiotics. There was a more prominent reduction of Proteobacteria and Firmicutes with a higher abundance of Bacteroides after L. plantarum administration when compared with E. faecium. However, the abundance of Bacteroides after treatment with both probiotics was lower than the non-probiotics obese mice. Although L. plantarum reduced both beneficial bacteria; Firmicutes bacteria (mostly Gram-positive bacteria that prominently identified in healthy gut [74]), and pathogenic bacteria (Proteobacteria) in feces, the improved intestinal mucosal integrity indicates favorable outcomes. Indeed, gut barrier defect of obese mice with L. plantarum was less severe than E. faecium as determined by FITC-dextran assay. However, both probiotics were effectively reduced endotoxemia levels when compared with obese mice control and the level of endotoxin was not different between probiotics. The prominent reduction in serum FITC-dextran in L. plantarum-treated mice compared with E. faeciumadministered mice with the non-difference in endotoxin levels, implying the higher sensitivity of FITC-dextran assay over LPS on gut barrier determination. Perhaps, this sensitivity difference is due to the smaller size of Dextran $(4.4 \mathrm{kDa})$ when compared with LPS $(>50 \mathrm{kDa})[11]$.

\subsection{Both Lactobacillus plantarum and Enterococcus faecium attenuated saturated fatty acid- induced inflammation in enterocytes and macrophages}

Although the benefit on attenuation of gut dysbiosis (reduced Proteobacteria) by $L$. plantarum was more potent than E. faecium (possibly through the excretion of different active molecules), both probiotics attenuated obesity-induced intestinal inflammation as indicated by the lower cytokines from colon tissue compared with the non-probiotics group. To explore the molecular nature of immunomodulating substances in a conditioned medium against enterocytes, the enzyme neutralizing protocols on supernatant IL8 production were used because of the predominant supernatant IL-8 in Caco-2 cells [75]. As such, the anti-inflammatory property of the condition media from L. plantarum and E. faecium was neutralized only by amylase enzyme and by amylase and lipase enzymes, respectively, indicating the active molecules with polysaccharide and lipo-polysaccharide, respectively. The anti-inflammatory effect on enterocytes improved enterocyte integrity (TEER) which might be responsible for the less severe obesity-enhanced gut barrier defect in mice. Additionally, the anti-inflammatory effect of the media from both probiotics was not only against palmitic acid but also toward LPS and LPS plus palmitic acid, implying a broad effect against several stimuli which might be suitable for medication. Although purification of bioactive substances was not performed, our initial 
characterization supposes the well-known importance of the exopolysaccharide from probiotics on anti-inflammatory effect $[21,76,77]$. In parallel, the probiotic molecules also down-regulated the cholesterol absorption associated molecules in similar to several probiotic studies [78].

Additionally, the molecules from condition media from both probiotics were also similarly attenuated palmitic acid-induced macrophage inflammation possibly through an alteration in cell energy status. Among all regions in the body, the intestine is the largest pool of macrophages which play a critical role in intestinal inflammation [79] and the attenuation of macrophage pro-inflammatory responses might be responsible for the less severe obesity-induced gut inflammation after probiotics administration. Here, the condition media from both probiotics down-regulate glycolysis activity, the main energy utilization for cytokine production [80]. Although the correlation between glycolysis and exopolysaccharide production from bacteria and the direct impact of probiotics on host enterocyte is mentioned $[81,82]$, data on the direct influence of exopolysaccharide against host cell energy status are still less and the extraction of exopolysaccharide for the new anti-inflammatory drug is interesting. More studies on this are warranted.

\section{Conclusions}

L. plantarum showed a more potent anti-obesity property than E. faecium possibly through the pre-dominant attenuation on dysbiosis (reduction in pathogenic Proteobacteria in feces) and gut barrier defect. Although both probiotics attenuated inflammation in enterocytes and macrophages, possibly through exopolysaccharide, that is interesting to use as a new anti-inflammatory treatment, L. plantarum might be more proper for use for anti-obesity concerning possible pathogenesis of E. faecium.

Author Contributions: Conceptualization, K.P. and T.O.; methodology, A.L. and T.O.; software, T.L., L.W., N.S. and N.N.; validation, T.O., A.L. and K.P.; formal analysis, T.O., N.S. and N.N.; investigation, T.O., S.K., T.L., L.W., N.S., N.N., K.J. and A.L.; resources, K.P.; data curation, T.O., K.J., T.L., L.W., N.S., N.N. and S.K.; writing-original draft preparation, T.O. and A.L.; writing-review and editing, T.O., A.L. and K.P.; visualization, A.L. and S.K.; supervision, K.P.; project administration, K.P. and K.J.; funding acquisition, K.P. All authors have read and agreed to the published version of the manuscript.

Funding: This research was funded by Thailand Research Fund, grant number RDG6150124; Thailand Government Fund, grant number RSA6080023; Chulalongkorn University Fundamental Fund 2565, grant number CU_FRB65_Food (1)_020_30_01; and National Research Council of Thailand, grant number NRCT5-RGJ63001 and NRCT-N41A640076 with NSRF via the Program Management Unit for Human Resources \& Institutional Development, Research and Innovation. T.O. and K.P. were funded by the Second Century Fund (C2F), Chulalongkorn University.

Institutional Review Board Statement: The protocol of animal care and use was approved by the Institutional Animal Care and Use Committee of the Faculty of Medicine, Chulalongkorn University, Bangkok, Thailand (SST 025/2563) in compliance with US National Institutes of Health standards.

Acknowledgments: The authors thank Dr. Puey Ounjai and Dr. Kamon Chaiyasit for their academic and administrative supports.

Conflicts of Interest: The authors declare no conflict of interest. The funders had no role in the design of the study; in the collection, analyses, or interpretation of data; in the writing of the manuscript, or in the decision to publish the results. 
1. Allison, D. B., Fontaine, K. R., Manson, J. E., Stevens, J. \& VanItallie, T. B. Annual deaths attributable to obesity in the United States. JAMA 1999, 282, 1530-1538, doi:10.1001/jama.282.16.1530.

2. Ross, P. A., Newth, C. J., Leung, D., Wetzel, R. C. \& Khemani, R. G. Obesity, and Mortality Risk in Critically Ill Children. Pediatrics 2016, 137, e20152035, doi:10.1542/peds.2015-2035.

3. Kolyva, A. S. et al. The role of obesity in the immune response during sepsis. Nutr Diabetes 2014, 4, e137, doi:10.1038/nutd.2014.34.

4. Singer, G., Stokes, K. Y., Terao, S. \& Granger, D. N. Sepsis-induced intestinal microvascular and inflammatory responses in obese mice. Shock 2009, 31, 275-279, doi:10.1097/SHK.0b013e3181834ab3.

5. Fruhbeck, G., Catalan, V., Rodriguez, A. \& Gomez-Ambrosi, J. Adiponectin-leptin ratio: A promising index to estimate adipose tissue dysfunction. Relation with obesity-associated cardiometabolic risk. Adipocyte 2018, 7, 57-62, doi:10.1080/21623945.2017.1402151.

6. Jaroonwitchawan, T. et al. Dysregulation of Lipid Metabolism in Macrophages Is Responsible for Severe Endotoxin Tolerance in FcgRIIB-Deficient Lupus Mice. Frontiers in Immunology 2020, 11, 959, doi:10.3389/fimmu.2020.00959.

7. McArdle, M. A., Finucane, O. M., Connaughton, R. M., McMorrow, A. M. \& Roche, H. M. Mechanisms of obesity-induced inflammation and insulin resistance: insights into the emerging role of nutritional strategies. Frontiers in Endocrinology 2013, 4, 52.

8. Eppensteiner, J. et al. Damage- and pathogen-associated molecular patterns play differential roles in late mortality after critical illness. JCI Insight 2019, 4, doi:10.1172/jci.insight.127925.

9. Chait, A. \& Kim, F. Saturated fatty acids and inflammation: who pays the toll? Arterioscler Thromb Vasc Biol 2010, 30, 692-693, doi:10.1161/ATVBAHA.110.203984.

10. Ondee, T. et al. Lactobacillus acidophilus LA5 improves saturated fat-induced obesity mouse model through the enhanced intestinal Akkermansia muciniphila. Sci Rep 2021, 11, 6367, doi:10.1038/s41598-021-85449-2.

11. Amornphimoltham, P., Yuen, P. S. T., Star, R. A. \& Leelahavanichkul, A. Gut Leakage of Fungal-Derived Inflammatory Mediators: Part of a Gut-Liver-Kidney Axis in Bacterial Sepsis. Dig Dis Sci 2019, 64, 2416-2428, doi:10.1007/s10620-019-05581-y.

12. Nagpal, R. et al. Obesity-Linked Gut Microbiome Dysbiosis Associated with Derangements in Gut Permeability and Intestinal Cellular Homeostasis Independent of Diet. J Diabetes Res 2018, 3462092, doi:10.1155/2018/3462092.

13. Heisel, T. et al. High-Fat Diet Changes Fungal Microbiomes and Interkingdom Relationships in the Murine Gut. $m S p h e r e$ 2017, 2, doi:10.1128/mSphere.00351-17.

14. Murphy, E. A., Velazquez, K. T. \& Herbert, K. M. Influence of high-fat diet on gut microbiota: a driving force for chronic disease risk. Curr Opin Clin Nutr Metab Care 2015, 18, 515-520, doi:10.1097/MCO.0000000000000209.

15. Leelahavanichkul, A. et al. Gastrointestinal Leakage Detected by Serum (1-->3)-beta-D-Glucan in Mouse Models and a Pilot Study in Patients with Sepsis. Shock 2016, 46, 506-518, doi:10.1097/SHK.0000000000000645.

16. Zhang, Q. et al. Clinicopathological correlation of keratinocyte growth factor and matrix metalloproteinase-9 expression in human gastric cancer. Tumori 2015, 101, 566-571, doi:10.5301/tj.5000367.

17. Scaldaferri, F. et al. Gut microbial flora, prebiotics, and probiotics in IBD: their current usage and utility. Biomed Res Int 2013, 435268, doi:10.1155/2013/435268.

18. Hager, C. L. \& Ghannoum, M. A. The mycobiome: Role in health and disease, and as a potential probiotic target in gastrointestinal disease. Dig Liver Dis 2017, 49, 1171-1176, doi:10.1016/j.dld.2017.08.025.

19. Zuo, T. \& Ng, S. C. The Gut Microbiota in the Pathogenesis and Therapeutics of Inflammatory Bowel Disease. Front Microbiol 2018, 9, 2247, doi:10.3389/fmicb.2018.02247.

20. Hosono, A. et al. Characterization of a water-soluble polysaccharide fraction with immunopotentiating activity from Bifidobacterium adolescentis M101-4. Biosci Biotechnol Biochem 1997, 61, 312-316, doi:10.1271/bbb.61.312.

21. Wu, M. H. et al. Exopolysaccharide activities from probiotic bifidobacterium: Immunomodulatory effects (on J774A.1 macrophages) and antimicrobial properties. Int J Food Microbiol 2010, 144, 104-110, doi:10.1016/j.ijfoodmicro.2010.09.003.

22. Surayot, U. et al. Exopolysaccharides from lactic acid bacteria: structural analysis, molecular weight effect on immunomodulation. Int J Biol Macromol 2014, 68, 233-240, doi:10.1016/j.ijbiomac.2014.05.005.

23. Gao, K. et al. Immunomodulation and signaling mechanism of Lactobacillus rhamnosus GG and its components on porcine intestinal epithelial cells stimulated by lipopolysaccharide. J Microbiol Immunol Infect 2017, 50, 700-713, doi:10.1016/j.jmii.2015.05.002.

24. Panpetch, W. et al. Lactobacillus rhamnosus L34 Attenuates Gut Translocation-Induced Bacterial Sepsis in Murine Models of Leaky Gut. Infect Immun 2018, 86, doi:10.1128/IAI.00700-17.

25. Kathrani, A., Larsen, J. A., Kass, P. H. \& Fascetti, A. J. Effect of short-term probiotic Enterococcus faecium SF68 dietary supplementation in overweight and obese cats without comorbidities. Vet Rec Open 2016, 3, e000164, doi:10.1136/vetreco-2015-000164.

26. Ghosh, N., Wood, M. F. \& Vitkin, I. A. Mueller matrix decomposition for extraction of individual polarization parameters from complex turbid media exhibiting multiple scattering, optical activity, and linear birefringence. J Biomed Opt 2008, 13, 044036, doi:10.1117/1.2960934.

27. Qiao, H. et al. Assessment of the physicochemical properties and bacterial composition of Lactobacillus plantarum and Enterococcus faecium-fermented Astragalus membranaceus using single molecule, real-time sequencing technology. Sci Rep 2018, 8, 11862, doi:10.1038/s41598-018-30288-x. 
28. Hanchi, H., Mottawea, W., Sebei, K. \& Hammami, R. The Genus Enterococcus: Between Probiotic Potential and Safety Concerns-An Update. Front Microbiol 2018, 9, 1791, doi:10.3389/fmicb.2018.01791.

29. Salminen, M. K. et al. Lactobacillus Bacteremia, Clinical Significance, and Patient Outcome, with Special Focus on Probiotic L. Rhamnosus GG. Clin Infect Dis 2004, 38, 62-69.

30. Lewin-Epstein, O. \& Hadany, L. Host-microbiome coevolution can promote cooperation in a rock-paper-scissors dynamics. Proc Biol Sci 2020, 287, 20192754, doi:10.1098/rspb.2019.2754.

31. Shapira, M. Gut Microbiotas and Host Evolution: Scaling Up Symbiosis. Trends Ecol Evol 2016, 31, 539-549, doi:10.1016/j.tree.2016.03.006.

32. Pratchayasakul, W. et al. Effects of high-fat diet on insulin receptor function in rat hippocampus and the level of neuronal corticosterone. Life Sci 2011, 88, 619-627, doi:10.1016/j.lfs.2011.02.003.

33. Sae-khow, K. et al. Pathogen-Associated Molecules from Gut Translocation Enhance Severity of Cecal Ligation and Puncture Sepsis in Iron-Overload $\beta$-Thalassemia Mice. J Inflamm Res 2020, 13, 719-735, doi:10.2147/JIR.S273329.

34. Thim-uam, A. et al. Leaky-gut enhanced lupus progression in the Fc gamma receptor-IIb deficient and pristane-induced mouse models of lupus. Sci Rep 2020, 10, 777, doi:10.1038/s41598-019-57275-0.

35. Visitchanakun, P. et al. Gut leakage enhances sepsis susceptibility in iron-overloaded $\beta$-thalassemia mice through macrophage hyperinflammatory responses. Am J Physio Gastrointestinal Liver Physiol 2020, 318, G966-G979, doi:10.1152/ajpgi.00337.2019.

36. Savari, F., Mard, S. A., Badavi, M., Rezaie, A. \& Gharib-Naseri, M. K. A new method to induce nonalcoholic steatohepatitis (NASH) in mice. BMC Gastroenterol 2019, 19, 125, doi:10.1186/s12876-019-1041-x.

37. Urai, T. et al. The relationship between cutaneous wounds made on obese mice or those with decreased body weight and serum leptin level. Health 2016, 8, 1015.

38. Klaikeaw, N., Wongphoom, J., Werawatganon, D., Chayanupatkul, M. \& Siriviriyakul, P. Anti-inflammatory and anti-oxidant effects of aloe vera in rats with non-alcoholic steatohepatitis. World J Hepatol 2020, 12, 363-377, doi:10.4254/wjh.v12.i7.363.

39. Jomehzadeh, N., Javaherizadeh, H., Amin, M., Rashno, M. \& Teimoori, A. Quantification of Intestinal Lactobacillus Species in Children with Functional Constipation by Quantitative Real-Time PCR. Clin Exp Gastroenterol 2020, 13, 141-150, doi:10.2147/CEG.S250755.

40. Sedgley, C. M. et al. Quantitative real-time PCR detection of oral Enterococcus faecalis in humans. Arch Oral Biol 2005, 50, 575583, doi:10.1016/j.archoralbio.2004.10.017.

41. Issara-Amphorn, J., Somboonna, N., Pisitkun, P., Hirankarn, N. \& Leelahavanichkul, A. Syk inhibitor attenuates inflammation in lupus mice from FcgRIIb deficiency but not in pristane induction: the influence of lupus pathogenesis on the therapeutic effect. Lupus 2020, 29, 1248-1262.

42. Schloss, P. D. et al. Introducing mothur: open-source, platform-independent, community-supported software for describing and comparing microbial communities. Appl Environ Microbiol 2009, 75, 7537-7541, doi:10.1128/AEM.01541-09.

43. Bulan, D. E. et al. Spatial and Seasonal Variability of Reef Bacterial Communities in the Upper Gulf of Thailand. Front Mar Sci 2018, 5, 441, doi:10.3389/fmars.2018.00441.

44. Segata, N. et al. Metagenomic biomarker discovery and explanation. Genome Biol 2011, 12, R60, doi:10.1186/gb-2011-12-6-r60.

45. Zhong, W., McClain, C. J., Cave, M., Kang, Y. J. \& Zhou, Z. The role of zinc deficiency in alcohol-induced intestinal barrier dysfunction. Am J Physiol Gastrointest Liver Physiol 2010, 298, G625-633, doi:10.1152/ajpgi.00350.2009.

46. Visitchanakun, P. et al. Increased susceptibility to dextran sulfate-induced mucositis of iron-overload beta-thalassemia mice, another endogenous cause of septicemia in thalassemia. Clin Sci (Lond) 2021, 135, 1467-1486, doi:10.1042/CS20210328.

47. Panpetch, W. et al. Candida Administration Worsens Uremia-Induced Gut Leakage in Bilateral Nephrectomy Mice, an Impact of Gut Fungi and Organismal Molecules in Uremia. mSystems 2021, 6, doi:10.1128/mSystems.01187-20.

48. Panpetch, W. et al. Candida Administration Worsens Cecal Ligation and Puncture-Induced Sepsis in Obese Mice Through Gut Dysbiosis Enhanced Systemic Inflammation, Impact of Pathogen-Associated Molecules From Gut Translocation and Saturated Fatty Acid. Front Immunol 2020, 11, 561652, doi:10.3389/fimmu.2020.561652.

49. Bhunyakarnjanarat, T. et al. Prominent Indomethacin-Induced Enteropathy in Fcgriib Defi-cient lupus Mice: An Impact of Macrophage Responses and Immune Deposition in Gut. Int J Mol Sci 2021, 22, doi:10.3390/ijms22031377.

50. Udompornpitak, K. et al. Lipopolysaccharide-Enhanced Responses against Aryl Hydrocarbon Receptor in FcgRIIb-Deficient Macrophages, a Profound Impact of an Environmental Toxin on a Lupus-Like Mouse Model. Int J Mol Sci 2021, 22, doi:10.3390/ijms22084199.

51. Gadelha, C. \& Bezerra, A. N. Effects of probiotics on the lipid profile: systematic review. J Vasc Bras 2019, 18, e20180124, doi:10.1590/1677-5449.180124.

52. Park, H.-E., Do, K.-H., Jeong, J.-W., Jung, Y.-H. \& Lee, W.-K. Cholesterol and Visceral Fat Lowering Effects of Combined Lactic Acid Bacteria (Lactobacillus casei WK3, Enterococcus faecium WK5, Bifidobacterium longum WK9, and Lactobacillus plantarum K-1) in High Fat Diet-Fed C57BL/6J Mice. Journal of the Korean Society of Food Science and Nutrition 2018, 47, 1210-1216, doi:10.3746/jkfn.2018.47.12.1210.

53. Mazloom, K., Siddiqi, I. \& Covasa, M. Probiotics: How Effective Are They in the Fight against Obesity? Nutrients 2019, 11, doi:10.3390/nu11020258.

54. Panpetch, W. et al. Candida Administration Worsens Cecal Ligation and Puncture-Induced Sepsis in Obese Mice Through Gut Dysbiosis Enhanced Systemic Inflammation, Impact of Pathogen-Associated Molecules From Gut Translocation and Saturated Fatty Acid. Front Immunol 2020, 11, doi:10.3389/fimmu.2020.561652. 
55. Panpetch, W. et al. Additional Candida albicans administration enhances the severity of dextran sulfate solution induced colitis mouse model through leaky gut-enhanced systemic inflammation and gut-dysbiosis but attenuated by Lactobacillus rhamnosus L34. Gut Microbes 2020, 11, 465-480.

56. Tseng, C. H. \& Wu, C. Y. The gut microbiome in obesity. J Formos Med Assoc 2019, 118 Suppl 1, S3-S9, doi:10.1016/j.jfma.2018.07.009.

57. Goldstein, E. J., Citron, D. M., Peraino, V. A. \& Cross, S. A. Desulfovibrio desulfuricans bacteremia and review of human Desulfovibrio infections. J Clin Microbiol 2003, 41, 2752-2754, doi:10.1128/jcm.41.6.2752-2754.2003.

58. Zheng, H. et al. Modulation of Gut Microbiome Composition and Function in Experimental Colitis Treated with Sulfasalazine. Front Microbiol 2017, 8, 1703, doi:10.3389/fmicb.2017.01703.

59. Estadella, D. et al. Lipotoxicity: effects of dietary saturated and transfatty acids. Mediators Inflamm 2013, 137579, doi:10.1155/2013/137579.

60. Su, Y. R. et al. High-Fat Diet Aggravates the Intestinal Barrier Injury via TLR4-RIP3 Pathway in a Rat Model of Severe Acute Pancreatitis. Mediators Inflamm 2019, 2512687, doi:10.1155/2019/2512687.

61. Cristofori, F. et al. Anti-Inflammatory and Immunomodulatory Effects of Probiotics in Gut Inflammation: A Door to the Body. Front Immunol 2021, 12, 578386, doi:10.3389/fimmu.2021.578386.

62. Viola, A., Munari, F., Sanchez-Rodriguez, R., Scolaro, T. \& Castegna, A. The Metabolic Signature of Macrophage Responses. Front Immunol 2019, 10, 1462, doi:10.3389/fimmu.2019.01462.

63. Ellulu, M. S., Patimah, I., Khaza'ai, H., Rahmat, A. \& Abed, Y. Obesity and inflammation: the linking mechanism and the complications. Arch Med Sci 2017, 13, 851-863, doi:10.5114/aoms.2016.58928.

64. Jurgonski, A., Juskiewicz, J. \& Zdunczyk, Z. A high-fat diet differentially affects the gut metabolism and blood lipids of rats depending on the type of dietary fat and carbohydrate. Nutrients 2014, 6, 616-626, doi:10.3390/nu6020616.

65. Chen, L., Li, H., Li, J., Chen, Y. \& Yang, Y. Lactobacillus rhamnosus GG treatment improves intestinal permeability and modulates microbiota dysbiosis in an experimental model of sepsis. Int J Mol Med 2019, 43, 1139-1148, doi:10.3892/ijmm.2019.4050.

66. Shin, N. R., Whon, T. W. \& Bae, J. W. Proteobacteria: microbial signature of dysbiosis in gut microbiota. Trends Biotechnol 2015, 33, 496-503, doi:10.1016/j.tibtech.2015.06.011.

67. Bloom, S. M. et al. Commensal Bacteroides species induce colitis in host-genotype-specific fashion in a mouse model of inflammatory bowel disease. Cell Host Microbe 2011, 9, 390-403, doi:10.1016/j.chom.2011.04.009.

68. Boutagy, N. E., McMillan, R. P., Frisard, M. I. \& Hulver, M. W. Metabolic endotoxemia with obesity: Is it real and is it relevant? Biochimie 2016, 124, 11-20, doi:10.1016/j.biochi.2015.06.020.

69. Zhang, C. et al. Structural resilience of the gut microbiota in adult mice under high-fat dietary perturbations. ISME J 2012, 6 , 1848-1857, doi:10.1038/ismej.2012.27.

70. Hildebrandt, M. A. et al. High-fat diet determines the composition of the murine gut microbiome independently of obesity. Gastroenterology 2019, 137, 1716-1724 e1711-1712, doi:10.1053/j.gastro.2009.08.042.

71. Panpetch, W. et al. Additional Candida albicans administration enhances the severity of dextran sulfate solution induced colitis mouse model through leaky gut-enhanced systemic inflammation and gut-dysbiosis but attenuated by Lactobacillus rhamnosus L34. Gut Microbes 2019, 1-16, doi:10.1080/19490976.2019.1662712.

72. Panpetch, W. et al. Oral administration of live-or heat-killed Candida albicans worsened cecal ligation and puncture sepsis in a murine model possibly due to an increased serum $(1 \rightarrow 3)-\beta$-D-glucan. PloS One 2017, 12, e0181439.

73. Kobyliak, N. et al. Probiotics in prevention and treatment of obesity: a critical view. Nutr Metab (Lond) 2016, 13, 14, doi:10.1186/s12986-016-0067-0.

74. Rinninella, E. et al. What is the healthy gut microbiota composition? A changing ecosystem across age, environment, diet, and diseases. Microorganisms 2019, 7, 14.

75. Eftang, L. L., Esbensen, Y., Tannæs, T. M., Bukholm, I. R. \& Bukholm, G. Interleukin-8 is the single most up-regulated gene in whole genome profiling of $\mathrm{H}$. pylori exposed gastric epithelial cells. BMC Microbiology 2021, 129.

76. Ayyash, M. et al. Exopolysaccharide produced by potential probiotic Enterococcus faecium MS79: Characterization, bioactivities and rheological properties influenced by salt and pH. Lwt 2020, 131, doi:10.1016/j.lwt.2020.109741.

77. Kanmani, P. et al. Synthesis and functional characterization of antibiofilm exopolysaccharide produced by Enterococcus faecium MC13 isolated from the gut of fish. Appl Biochem Biotechnol 2013, 169, 1001-1015, doi:10.1007/s12010-012-0074-1.

78. Yoon, H. S. et al. Reduction in cholesterol absorption in Caco-2 cells through the down-regulation of Niemann-Pick C1-like 1 by the putative probiotic strains Lactobacillus rhamnosus BFE5264 and Lactobacillus plantarum NR74 from fermented foods. Int J Food Sci Nutr 2013, 64, 44-52, doi:10.3109/09637486.2012.706598.

79. Wang, S., Ye, Q., Zeng, X. \& Qiao, S. Functions of Macrophages in the Maintenance of Intestinal Homeostasis. J Immunol Res 2019, 1512969, doi:10.1155/2019/1512969.

80. Yu, Q. et al. Regulations of Glycolytic Activities on Macrophages Functions in Tumor and Infectious Inflammation. Front Cell Infect Microbiol 2020, 10, 287, doi:10.3389/fcimb.2020.00287 (2020).

81. Ramos, A., Boels, I. C., de Vos, W. M. \& Santos, H. Relationship between glycolysis and exopolysaccharide biosynthesis in Lactococcus lactis. Appl Environ Microbiol 2001, 67, 33-41, doi:10.1128/AEM.67.1.33-41.2001.

82. Xu, Y. et al. Function of Akkermansia muciniphila in Obesity: Interactions With Lipid Metabolism, Immune Response and Gut Systems. Front Microbiol 2020, 11, 219, doi:10.3389/fmicb.2020.00219. 